\title{
Improved Yield and Data Quality in Atom Probe Tomography
}

\author{
R.M. Ulfig, E. Oltman, D.R. Lenz, T.R. Payne, T.J. Prosa, and D.J. Larson
}

Cameca Instruments Inc., 5500 Nobel Drive, Madison, WI 53719-1193

Problems with low sample yield or low data quality have sometimes plagued new applications for atom probe tomography (APT). For example, early in the efforts to run silicon oxide regions greater than $10 \mathrm{~nm}$ thick, they were considered unsuitable due to a combination of low sample yield, poor mass-spectral quality and three-dimensional reconstructed data quality [1]. Recent hardware and software improvements available with the LEAP $4000 \mathrm{X}^{\circledR}$ have resulted in higher yield and higher data quality. Improvements include a shorter-wavelength laser, a more tightly focused laser spot, advanced evaporation response algorithms, live-time display of charge-state ratio and noise level, drift compensation, and constant flux mode with areal evaporation rate control. The combination of all of these features has made previously ineffective applications tenable. This work reviews these features with respect to a $120 \mathrm{~nm}$ thick silicon dioxide layer that yielded zero successful runs previously, but has recently been analyzed with success and high data quality.

The new 355nm-wavelength laser of the LEAP 4000X enables a more tightly focused diffractionlimited spot and a shallower absorption depth. The result is a higher mass resolving power and signal-to-noise ratio due to a smaller heated volume (smaller illuminated region and the energy is deposited to lesser depth). The specimen tip thus heats and cools substantially faster [2]. Thick dielectrics, once very problematic, now can be analyzed with acceptable yield and high signal-tonoise ratio and spatial resolution [1], Fig. 1.

New evaporation-rate control algorithms implemented in the system firmware and software allow near-instantaneous response (before the next pulse) for adjustment of voltage and laser pulse amplitudes applied to the specimen. A dual-channel fast-response algorithm decreases voltage when a certain number of sequential pulses have each caused detected evaporation events and also will apply a conventional voltage drop in response to exceeding a user-selected evaporation rate. These measures lead to demonstrably higher specimen yields on materials with heterogeneous interfaces like this thick oxide application [1], Fig. 2.

Data collection from fracture-prone specimens or specimens requiring large volume analysis can require long acquisition times (12 hours or more) and subject the run to environmental (temperature) changes in all but the most well designed microscopy labs. Temperature changes and component drift (on the order of microns) can lead to misalignments among the specimen, the counter electrode, and the laser location. Intelligent auto alignment algorithms compensate for long-cycle changes and keep data quality high throughout the experiment. They also keep the specimen and local electrode alignment consistent from the start of the experiment regardless of operator experience or preference. Auto drift control, coupled with new algorithms that estimate the field of view of the analysis at all times, control specimen evaporation rates with a target ion flux [ions $/ \mathrm{nm}^{2} / \mathrm{sec}$ ] and maintain a constant evaporation field throughout the run regardless of variations in tip diameter. Additionally, limits can be set on maximum evaporation rates within regions of the detector. As new phases are exposed to the evaporating surface, they can lead to temporally and spatially changing ion flux as shown in Fig. 2. This can lead to excessive fields as the computer tries to increase evaporation rates which can cause specimen fracture or low signal-to-noise ratios. The new control algorithms adjust the global ion flux to compensate. 
Unfortunately, subtle differences in specimen geometry or laser focus, even with the same energy per pulse, can cause differences in data quality and compositional accuracy. New control software displays composition and data quality while monitoring the delivered energy per pulse. Simultaneous readout of a noise metric, composition and charge-state ratio (which is correlated with evaporation field) allows an operator to choose run conditions to meet identical charge-state ratio from one run to the next and therefore collect data under near-identical conditions. This is advantageous both for consistent experimental conditions, and for consistency in the required reconstruction parameters.

The suite of new software features for the LEAP 4000 platform improves data quality and increases sample yield. The shorter time to knowledge and the smaller numbers of samples required means a higher return on investment on both sample preparation systems and on the LEAP itself. The control software continues to evolve to give both new users and experienced experimentalists the simplicity and flexibility required to work on new applications. [3]

References

[1] T. J. Prosa et al., These Proceedings (2011).

[2] J.H. Bunton et al., Microsc. Microanal. 13 (6) (2007) 418-427.

[3] The authors would like to acknowledge the entire CAMECA Madison team for their contributions.
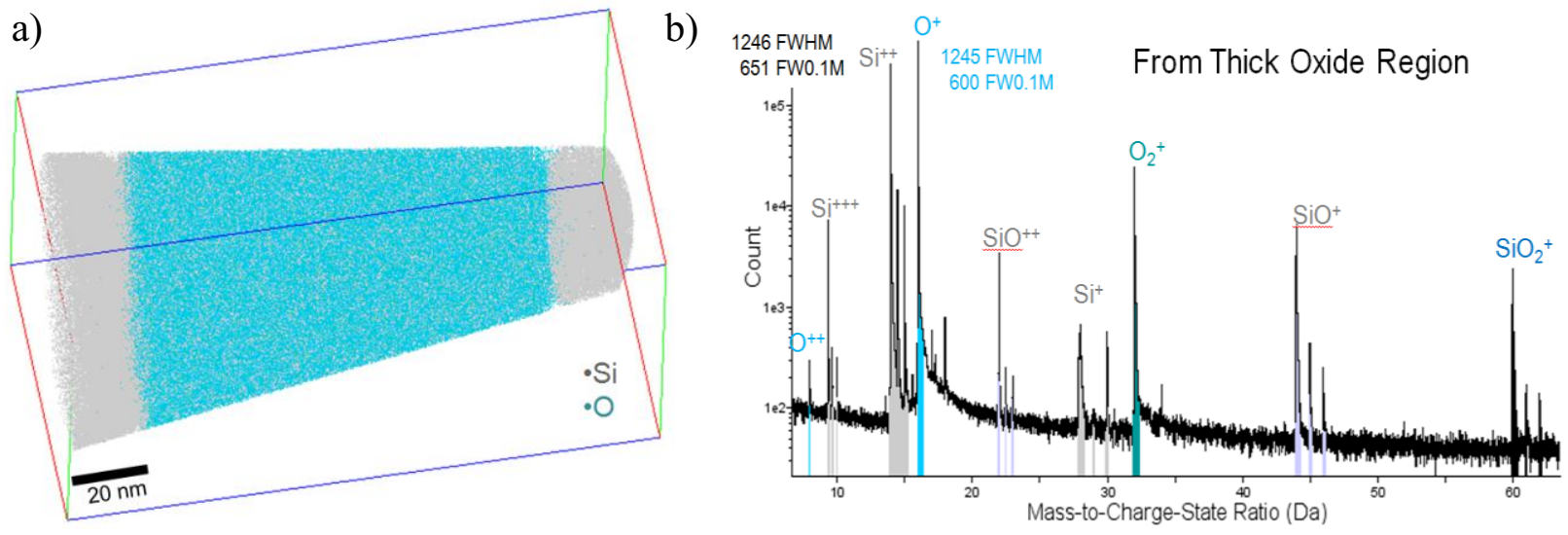

FIG. 1. a) High quality atom map of thick $\mathrm{SiO}_{2}$ film. b) Mass spectrum from thick oxide region exhibiting high mass resolving power and signal-to-noise ratio, even from within the oxide region.
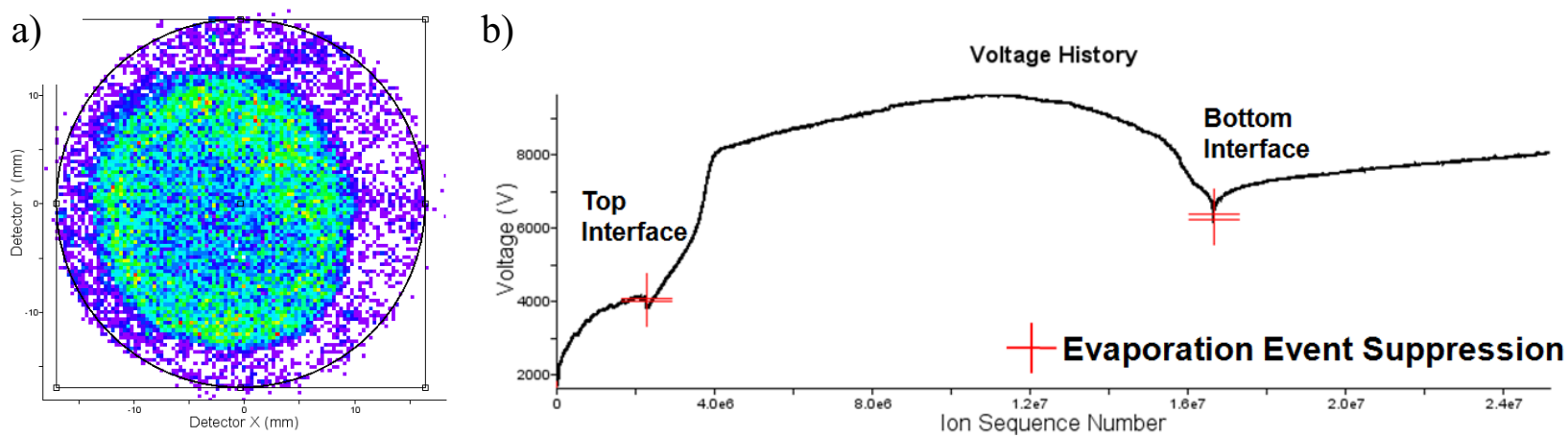

FIG. 2 a) Evaporation hit map from the top interface (Poly $\left.\mathrm{Si}_{\text {to }} \mathrm{SiO}_{2}\right)$ and b) voltage history. Local evaporation rates are detected and controlled with evaporation event responses noted at the interfaces of the top and bottom of the $\mathrm{SiO}_{2}$ layer maximize yield and data quality. 